\title{
EchoGéo
}

$34 \mid 2015$

Varia

\section{Mobilités socio-spatiales et production territoriale en Sénégambie}

Analyse à partir de la frontière Sénégal-Gambie

Mohamadou Mountaga Diallo

\section{CpenEdition}

Journals

Édition électronique

URL : https://journals.openedition.org/echogeo/14411

DOI : 10.4000/echogeo.14411

ISSN : 1963-1197

Éditeur

Pôle de recherche pour l'organisation et la diffusion de l'information géographique (CNRS UMR 8586)

Référence électronique

Mohamadou Mountaga Diallo, « Mobilités socio-spatiales et production territoriale en Sénégambie », EchoGéo [En ligne], 34 | 2015, mis en ligne le 15 décembre 2015, consulté le 31 juillet 2021. URL http://journals.openedition.org/echogeo/14411 ; DOI : https://doi.org/10.4000/echogeo.14411

Ce document a été généré automatiquement le 31 juillet 2021.

EchoGéo est mis à disposition selon les termes de la licence Creative Commons Attribution - Pas d'Utilisation Commerciale - Pas de Modification 4.0 International (CC BY-NC-ND) 


\section{Mobilités socio-spatiales et production territoriale en Sénégambie}

Analyse à partir de la frontière Sénégal-Gambie

\section{Mohamadou Mountaga Diallo}

\section{Introduction}

1 La littérature sur la géographie des frontières ouest-africaines s'est appesantie pendant longtemps sur le rapport frontière-territoire étatique qu'elle a diversement apprécié. Les réflexions ont porté dans 2 directions. Tout d'abord, sur la critique de l'artificialité des frontières qui sont, $\mathrm{du}$ fait de leur caractère arbitraire et exogène, responsables du sous-développement et des conflits territoriaux en Afrique (Barry, 1988 ; Kane, 2010). Et d'autre part sur la relativisation de l'artificialité en raison de la prise en compte, par les colonisateurs, des réalités locales et l'implication des pouvoirs africains dans la mise en place des frontières (Foucher, 2014 ; Lefebvre, 2015).

2 Les flux commerciaux transfrontaliers, dus à l'exploitation par les acteurs marchands des disparités fiscalo-douanières entre États bordiers, sont un important facteur de déstructuration et de désintégration (Badie, 1995 ; Bach, 1998 ; Meagher, 2003). En effet, le dynamisme des flux d'échanges transfrontaliers entraîne le développement de "périphéries nationales " (Igué, 1995) qui gardent une certaine autonomie vis-à-vis des lois qui régissent le territoire national. Ces espaces périphériques s'imposent par leur rôle économique et social aux État-nations limitrophes avec l'usage de plusieurs monnaies dont le cours est déterminé par un marché local de change parallèle. Cette autonomie et la forte connexion spatiale entre espaces frontaliers, par-delà les frontières, remettent en cause l'efficacité du contrôle étatique, portant ainsi un sérieux coup à l'intégrité territoriale (Igué, 1995 ; Stary, 1995 ; Dubresson et Raison, 2003).

3 L'idée de "déterritorialisation ", c'est-à-dire de désintégration des territoires étatiques est cependant contestée par certains auteurs dont Karine Bennafla (2002). Elle 
considère les flux transfrontaliers plutôt comme un facteur d'intégration territoriale. En effet, la fugacité des constructions spatiales frontalières (marchés frontaliers, villesentrepôts, petites villes) et l'absence d'un pouvoir politique transfrontalier qui gère la "périphérie transnationale», née de la dynamique des échanges, relativisent de tels processus de désintégration. D'ailleurs, la forte connexion entre les espaces frontaliers et les zones de l'intérieur (villes moyennes et capitales d'État) se traduit par la consolidation du puzzle territorial national.

4 Au-delà du débat contradictoire sur l'artificialité des frontières et l'effet des flux transfrontaliers sur le territoire national, le rapport frontière-territoire ne peut-il être appréhendé dans une autre perspective?

Depuis plus d'une vingtaine d'années (1990-2014), de nombreux travaux de géographes illustrent le changement de perspective dans l'étude des frontières africaines. Ils s'inscrivent dans une approche zonale avec un accent sur les effets socio-spatiaux des frontières et les liens transfrontaliers (Igué, 1995 ; Bennafla, 2002 ; Enda Diapol, 2007 ; Walther, 2008 ; 2007 ; Kane A.F, 2010 ; Foucher, 2014 ; Diallo, 2014). Ces études montrent que les zones frontalières fonctionnent en réseau, elles ne connaissent pas de véritables ruptures spatiales. Ces frontières imposées rapprochent les populations locales plus qu'elles ne les éloignent en permettant des échanges transfrontaliers. Elles sont vues comme une ressource car « tout un monde vit des asymétries frontalières (marchands et transporteurs, douaniers et militaires) exprimées par des différentiels de change, d'offre, de norme, de prix » (Foucher, 2014, p. 36).

Cependant, ces études insistent plus sur les flux commerciaux et économiques ainsi que sur le rôle des espaces, notamment urbains, dans l'organisation des échanges. C'est ce que note Olivier Walther: "longtemps cantonnées à la critique de l'artificialité des limites coloniales, les contributions des géographes à la théorie des frontières sahéliennes s'attachent aujourd'hui à montrer comment ces espaces peuvent constituer des environnements potentiellement fertiles pour les activités productives et marchandes...Les géographes engagés dans la réflexion sur les frontières ouestafricaines en sont venus à considérer ces espaces comme une partie centrale de réseaux économiques fondés sur un certain nombre de centres urbains " (Walther, 2007, p. 34).

S'inscrivant dans la même dynamique, cet article tente d'aller plus loin en questionnant le rapport des populations à la frontière, à travers notamment les processus de construction de territoires transfrontaliers. Il se fonde sur l'hypothèse selon laquelle les acteurs se construisent une territorialité transfrontalière à travers leurs mobilités socio-spatiales liées à des motivations non seulement marchandes mais aussi et surtout sociale, culturelle, religieuse et économique. Les mobilités consécutives à l'exploitation des différentiels frontaliers entraînent une mise en relation des lieux conduisant ainsi les acteurs de la mobilité à se créer des espaces vécus transfrontaliers. Ces territoires se construisent à partir de pratiques spatiales et de représentations des acteurs de la mobilité. Ici, le territoire n'est pas celui de l'État, une entité politique délimitée par des frontières géopolitiques (Badie, 1995), mais plutôt un produit social, aux limites floues et mouvantes (Di Méo, 2001; Bonnemaison, 1981), œuvre de la mobilité des acteurs locaux consécutive à l'effet-frontière. La frontière est ici lien et couture. Ces territoires transfrontaliers construits et donc fonctionnels revêtent, dans le contexte africain et sénégambien en particulier, une troisième dimension, celle de la gouvernance. En effet, espaces partagés et de fortes solidarités intercommunautaires, ces territoires transfrontaliers sont cependant l'objet de compétitions et de conflits qui sont 
aujourd'hui pris en charge par des acteurs locaux ou non à travers diverses initiatives de coopération transfrontalière s'inscrivant dans une démarche de gouvernance territoriale.

8 La région de la Sénégambie sur laquelle nous mettons ici l'accent en particulier constitue un terrain pertinent pour examiner cette hypothèse relative aux processus de construction de territorialités transfrontalières. La Sénégambie renvoie à ce vaste espace géographique constitué par les deux bassins du fleuve Sénégal et du fleuve Gambie. Du point de vue administratif, il couvre l'intégralité des États du Sénégal, de la Gambie et de la Guinée Bissau ainsi qu'une partie des États de la Mauritanie, du Mali et de la Guinée Conakry (Barry, 1988).

Illustration 1 - Localisation de la zone d'étude dans l'espace sénégambien

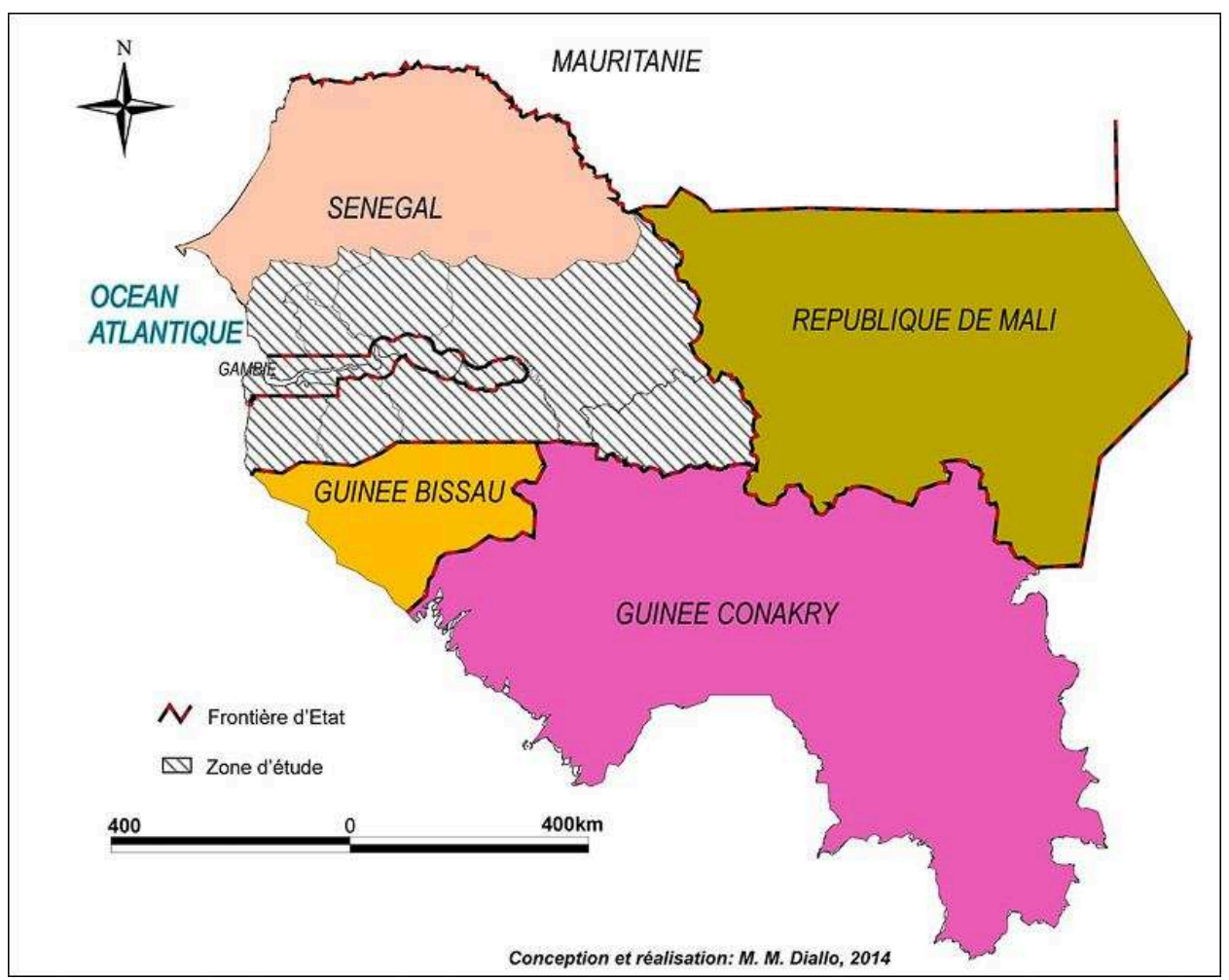

Auteur ; M.M. Diallo, 2014.

9 La région, qui a appartenu tour à tour à l'empire du Mali puis au Gabou, est marquée par une continuité géographico-sociale très forte (Enda Diapol, 2007 ; Kane, 2010). La densité du réseau hydrographique, les conditions pédologiques et pluviométriques offrent à la région un important potentiel permettant le développement d'une économie complémentaire fondée sur l'agriculture (arachide, coton, riz), l'élevage, la pêche et le commerce (Fanchette, 2001; Sidibé, 2003). Sur le plan social, même si la mise en place des frontières coloniales a entrainé l'émiettement politique de la région, les relations entre groupes sociaux ont permis le brassage des populations et la formation de plusieurs territoires socio-historiques comme le Fogny, le Kombo, le Sabakh, le Badibou, le Rip, le Saloum.

Sur le plan géopolitique, la Sénégambie est marquée par une instabilité à dimension transfrontalière voire régionale qui se nourrit, en partie, de la porosité des frontières et de la forte imbrication des sociétés. Depuis plus d'une vingtaine d'années, le conflit 
armé en Casamance, l'instabilité politique en Guinée Bissau et en Guinée Conakry, les tentatives avortées de coup d'État en Gambie et récemment la crise malienne affectent profondément la vie socioéconomique de la Sénégambie. La récente crise d'Ebola et la fermeture des frontières (Sénégal-Guinée Conakry et Guinée Bissau-Guinée Conakry) qui s'en est suivie en créant des tensions diplomatiques entre les États susmentionnées a révélé toute la dimension géopolitique des frontières en Sénégambie; la rapide diffusion de la maladie à virus Ebola est, en partie, liée à la porosité des frontières en Afrique de l'Ouest (Simon-Lorière et Lysaniuk, 2015). La région est, en outre, marquée par le développement de la criminalité transfrontalière, le trafic de drogue et de bois.

11 Ce contexte socioculturel, géoéconomique et géopolitique fait donc de la Sénégambie une région à forte mobilité humaine. Les populations sont très mobiles et la frontière, une ressource importante. Dès lors, des rapports socio-spatiaux émergent aboutissant à des processus complexes de construction de territorialités transfrontalières.

Cet article se fonde sur des résultats d'enquêtes menées sur la frontière sénégalogambienne, dans le cadre d'une thèse, entre juillet-août et septembre puis en novembre-décembre 2010, auprès de 360 chefs de ménage et 730 acteurs du commerce transfrontalier (marchands et acheteurs) ainsi que de nombreux entretiens semidirectifs avec divers acteurs de la vie frontalière. Dans une première partie, l'article aborde la mobilité transfrontalière dans ses diverses formes ainsi que les multiples facteurs qui la déterminent avant d'analyser dans un second temps comment les populations frontalières, à travers leurs diverses mobilités socio-spatiales, fabriquent des territoires sociaux et marchands. Ces territoires, appropriés et vécus, sont partagés par plusieurs acteurs de part et d'autre de la frontière et font parfois l'objet d'une âpre compétition au regard des enjeux qu'ils renferment (ressources naturelles, sécurité, migration, etc). Se pose ainsi la question de leur gouvernance par les acteurs concernés. Ceci amènera à aborder, enfin, la question de la gouvernance transfrontalière, très actuelle en Afrique de l'Ouest, dans un double contexte de décentralisation et de régionalisation ${ }^{1}$.

\section{Frontières et mobilités en Sénégambie}

En Sénégambie, la mise en place des frontières n'a pas partout tenu compte des réalités de la géographie humaine. Le choix des tracés frontaliers s'est traduit par la séparation de plusieurs groupes ethniques comme ailleurs en Afrique de l'Ouest et dans le reste du monde d'ailleurs. Car la mise en place des frontières africaines s'est appuyée sur des considérations politiques avec l'appui des autorités locales et non sur les réalités sociolinguistiques (Lefebvre, 2015) ${ }^{2}$. Les exemples des peuls entre le Sénégal et la Guinée Conakry, des Diola et Wolof entre la Gambie et le Sénégal, sont de bonnes illustrations de cet état de fait, conséquence de l'Histoire. Par ailleurs, les périodes coloniales et postcoloniales ont été marquées en Sénégambie par la mise en place de nouveaux États qui ont adopté des politiques économiques, fiscales et monétaires fort différentes. Ces disparités ainsi que les liens socioculturels entre groupes ethniques et les différences de niveau d'équipement entre les espaces frontaliers sénégambiens sont à l'origine d'une intense circulation humaine et marchande. La mobilité, dans l'espace sénégambien, tire largement profit de l'ouverture des frontières et d'une perméabilité matérialisées par la multiplicité des postes-frontières ${ }^{3}$. Les contrôles aux points de 
passages frontaliers sont peu intenses et les délais d'attente très courts voire inexistants sauf en cas de blocus et/ou de fermeture des frontières.

\section{Une intense mobilité humaine à travers des frontières poreuses}

14 Dans les espaces frontaliers sénégambiens, les mobilités transfrontalières sont intenses. Pendulaires, de courte et/ou de longue durée, ces mobilités sont liées à des raisons familiales, professionnelles, économiques ou encore sanitaires et scolaires.

Sur le plan familial, des dizaines de personnes traversent chaque jour la frontière pour rendre visite à des parents et/ou participer à des cérémonies socioculturelles. A Médina Sabakh, village sénégalais de 4672 habitants, $80 \%$ des personnes interrogées affirment que des membres de leur ménage traversent quotidiennement la frontière pour des raisons familiales. Cette mobilité est liée aux forts liens familiaux et à la forte solidarité intercommunautaire entre populations frontalières. Le tracé des frontières a séparé arbitrairement des populations appartenant aux mêmes groupes ethniques et aux mêmes familles. Par exemple, les populations de la zone transfrontalière constituée de l'arrondissement de Médina Sabakh (Sénégal) et du district du Upper Badibou (Gambie) appartenaient aux provinces du Sabakh et du Badibou qui faisaient partie du royaume du Rip. Ces deux provinces s'étendaient de part et d'autre de la frontière actuelle. Mais la mise en place de celle-ci, le 10 août 1889, a entraîné la séparation de ces populations qui ont maintenu cependant leurs relations sociales et culturelles.

Dans les espaces frontaliers sénégambiens, la mobilité est liée aussi à des raisons scolaires. Sur la frontière Nord sénégalo-gambienne, les établissements scolaires des villages sénégalais de Médina Sabakh, Passy Ngayène, Keur Ayib, Keur Moussa, reçoivent des élèves venant de la Gambie. A Farafenni, 27,3 \% des personnes interrogées affirment avoir des enfants qui étudient à Médina Sabakh. Le trajet inverse est fait quotidiennement par des enfants sénégalais qui ont été exclus du système scolaire et/ ou dont les parents ont une préférence pour l'enseignement anglophone. Dans la partie sud de la frontière, la scolarité transfrontalière est plutôt déterminée par le manque d'écoles dans les villages casamançais, qui oblige les parents à avoir recours aux écoles gambiennes pour la scolarisation de leurs enfants.

Par ailleurs, les populations frontalières ont recours aux établissements sanitaires situés de part et d'autre de la frontière. Ces déplacements s'inscrivent dans la logique des populations frontalières, qui utilisent la frontière en fonction de leurs besoins et des opportunités offertes. Ainsi, la sollicitation par les populations gambiennes des services de santé de Médina Sabakh s'explique par la qualité des soins offerts et la proximité de l'infrastructure sanitaire sénégalaise, alors que la fréquentation de l'hôpital de Farafenni par les Sénégalais est motivée par le coût des soins jugé moins onéreux que ceux proposés au Sénégal.

Les déplacements journaliers des populations frontalières se font aussi vers les marchés ruraux et urbains frontaliers. Les populations s'approvisionnement de part et d'autre des frontières et ce en fonction de la disponibilité des marchandises, du prix d'achat et de la proximité. Ainsi, le recours aux marchés gambiens est en partie motivé par l'éloignement des centres urbains sénégalais. Depuis Pata, village frontalier sénégalais, la ville sénégalaise la plus proche est Kolda, à $65 \mathrm{~km}$, alors que la ville gambienne de Brikama-Ba ne se trouve qu'à $9 \mathrm{~km}$. Ainsi, il est plus facile pour les habitants de Pata de s'approvisionner en Gambie qu'à Kolda. 

Les commerçants, très mobiles, fréquentent ces divers marchés où ils échangent produits primaires en provenance des campagnes sénégambiennes et/ou manufacturés importés du marché mondial. En Sénégambie, comme ailleurs en Afrique de l'Ouest (Walther, 2008 ; Walther et Retaillé, 2008), la circulation marchande transfrontalière est une continuité d'acteurs et de lieux. Des réseaux commerciaux fondés sur la foi religieuse et/ou l'appartenance géographico-sociale (origine géographique ou ethnique) organisent le commerce transfrontalier et régional. L'analyse de l'organisation du commerce peul de denrées alimentaires et du déploiement des marchands dans l'espace permet de noter une certaine continuité d'acteurs généralement organisé en réseau, du grossiste-transporteur et homme d'affaires situé à l'amont du réseau, au petit détaillant de l'aval, exerçant dans les quartiers urbains ou les villages aussi bien à l'échelle nationale qu'internationale. En effet, "plusieurs commerçants peuls contrôlent la distribution d'oignons ou de riz importés par le Sénégal ou la Gambie jusqu'à l'écoulement à Bissau. Un tel commerce se distingue du commerce en relais parce qu'il intègre l'ensemble de la filière de commercialisation et traduit une structuration avant tout communautaire» (Diapol, 2007, p. 71). 

s'appuient sur un réseau de solidarité fondé sur l'appartenance ethnique et géographique pour écouler leurs marchandises auprès des détaillants. À cet égard, la Haute Casamance (région de Kolda) constitue un exemple particulièrement illustratif. Fanchette (2001, p. 102) observe en effet «qu'à Kolda tous les grossistes ont des représentants dans les villages relais qui les informent des transactions intéressantes ou stockent leurs marchandises, des succursales dans les quartiers et des représentants dans les boutiques, chez les "tabliers" ou dans les cantines qui leur servent de paravent ».

Illustration 2 - Organisation de l'espace transfrontalier étudié

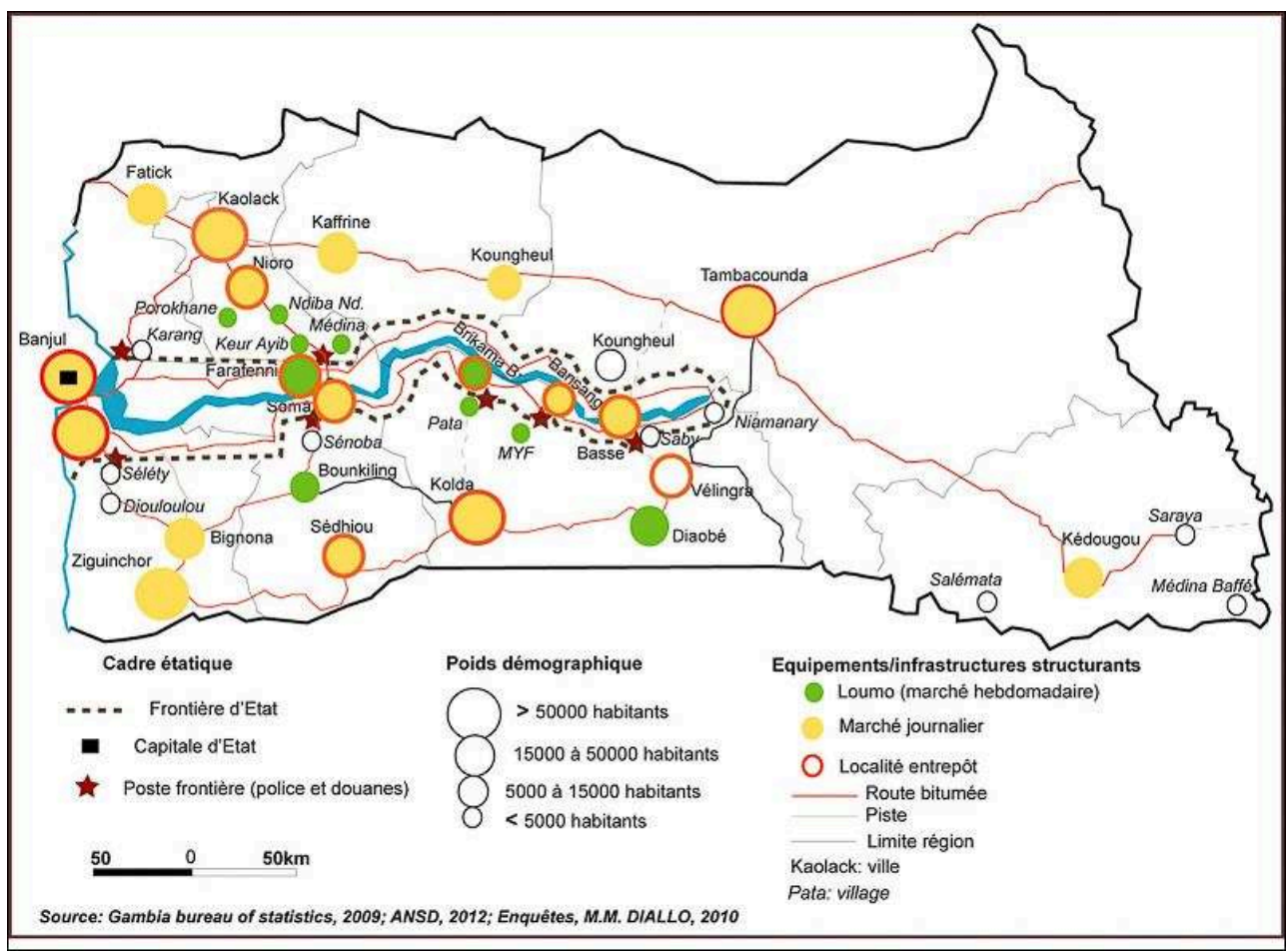

Sources : Gambia bureau of statistics, 2009 ; ANSD, 2012 ; enquêtes M.M. Diallo, 2010. 
Les échanges transfrontaliers s'appuient sur des places marchandes constituées de marchés quotidiens des centres urbains frontaliers, de villes entrepôts mais aussi de loumo. Ces espaces d'échange se sont développés en Sénégambie à partir des années 1980, dans un contexte de crise de l'État et de rareté de l'emploi formel, à la suite de l'application des programmes d'ajustement structurels imposés par les bailleurs de fonds (Fanchette, 2001 ; Ninot et al, 2002 ; Enda Diapol, 2007). transfrontaliers. C'est donc principalement à partir de ces espaces d'échanges que peut être appréciée la mobilité marchande en Sénégambie. Les loumo frontaliers sénégalais constituent un important centre d'écoulement des produits primaires pour les paysans gambiens. La grande majorité des paysans écoulant leurs produits agricoles au loumo de Médina Sabakh (Sénégal) sont des Gambiens du district frontalier du Upper Badibou. La forte fréquentation hebdomadaire du marché sénégalais par ces paysans est motivée par la possibilité d'accéder au franc CFA notamment, qui a plus de pouvoir d'achat que le dalassi gambien. De plus, le prix au producteur est plus intéressant à Médina Sabakh qu'en Gambie. Le kilogramme de mil et d'arachide par exemple était vendu, en 2010, respectivement à 135 et 150 FCFA au Sénégal contre 120 et 125 FCFA en Gambie.

8 En Gambie, de nombreux loumo frontaliers sont animés par des commerçants sénégalais venus des villages environnants et des villes proches. À Sare Bodjo, la majorité des commerçants du marché à bétail viennent de la région de Kolda. Sur la frontière Nord, le loumo de Farafenni est le principal centre d'écoulement des produits forestiers et maraîchers pour de nombreux paysans des villages sénégalais frontaliers. On y rencontre aussi des commerçants sénégalais de Nioro du Rip et de Kaolack venus pour vendre des produits manufacturés. Ces derniers profitent de leur présence pour s'approvisionner en produits destinés à la revente dans les centres urbains sénégalais tandis que certains marchands venus du monde rural achètent des produits manufacturés gambiens pour les revendre dans leurs villages. Ce mécanisme illustre la double fonction des loumo frontaliers qui sont pour les marchands à la fois des lieux d'écoulement et des centres d'approvisionnement en produits divers.

A côté des loumo, les villes frontalières constituent un des lieux importants de l'espace marchand. Elles sont des lieux d'approvisionnement, d'habitation et abritent des services indispensables à l'activité commerciale (banques, bureaux de change, etc). Ces différentes fonctions leur confèrent un rôle primordial dans l'activité marchande transfrontalière. Elles sont fréquentées par les acteurs marchands pour diverses raisons. La ville frontalière constitue d'abord un important centre d'approvisionnement pour les marchands. Les commerçants des marchés ruraux des localités frontalières de Médina Sabakh, Pata et Médina Yoro Foula dépendent ainsi fortement des marchés urbains respectifs de Farafenni, Brikama Ba et Bansang pour leur approvisionnement en produits manufacturés. La fréquentation périodique des marchés gambiens par les commerçants sénégalais s'explique par la disponibilité des produits recherchés, leur prix abordable et la proximité (qui réduit le coût du transport). Il faut préciser cependant que cet approvisionnement se fait dans la plupart des cas de manière frauduleuse, en marge des circuits officiels. En effet, il porte essentiellement sur des produits alimentaires de consommation courante (sucre, tomate, huile végétale) et manufacturés importés du marché mondial via le port de Banjul et dont l'entrée au Sénégal est soumise à une réglementation douanière. 
30 Ensuite, c'est dans les villes frontalières que logent la plupart des commerçants qui animent l'activité marchande transfrontalière. $45 \%$ des commerçants interrogés au loumo de Médina Yoro Foula (Sénégal) et $40 \%$ à Pata (Sénégal) viennent respectivement de Bansang et de Brikama-Ba, les deux villes gambiennes les plus proches de ces marchés. Dans ces villes sont installés les commerçants et parfois leurs familles. Ils y tiennent une boutique et/ou un magasin de stockage au marché central. C'est donc à partir de cette ville qu'ils sillonnent les différents loumo frontaliers tout au long de la semaine. La ville est donc partie intégrante de l'espace marchand aussi bien pour les commerçants qui y habitent que pour ceux qui s'y approvisionnent périodiquement.

31 Les populations frontalières de la Sénégambie sont donc très mobiles. Leur mobilité est déterminée par des motivations sociales, culturelles, religieuses, économiques et marchandes. Elle se fait quotidiennement et saisonnièrement et amène les populations à fréquenter divers espaces situés de part et d'autre des frontières d'État. De ce fait, ces mobilités tranfrontalières sont déterminantes dans la définition des espaces de vie des populations frontalières.

\section{Mobilités transfrontalières et construction territoriale}

Les mobilités transfrontalières qui se déploient à divers niveaux (villes et campagnes) amènent à s'interroger sur leur rôle dans la construction territoriale. Elles semblent jouer un rôle primordial dans la naissance de territoires différents de ceux servant de support à l'État-nation, c'est-à-dire les territoires politiques circonscrits à l'intérieur de frontières administratives. Cette construction territoriale, si elle est effective, soulève une question cruciale, celle de la gestion et de la gouvernance d'espaces non administratifs, ces territoires de la mobilité transfrontalière. Se pose dès lors la question de la coopération transfrontalière, très actuelle aujourd'hui en Afrique de l'Ouest.

\section{La production quotidienne des territoires par la mobilité socioéconomique}

Les acteurs socioéconomiques des espaces frontaliers, à travers leurs diverses mobilités quotidiennes, intègrent dans leur espace de vie divers lieux situés de part et d'autre de la frontière.

Le schéma ci-dessous illustre l'espace de vie de nombreuses populations frontalières. Pour des centaines d'habitants de la ville gambienne de Farafenni, le poste de santé de Médina Sabakh constitue le principal lieu de recours aux soins. Certains d'entre eux ont dans ce village sénégalais une partie de leur famille et leurs terres de culture. Ils y écoulent leurs produits agricoles et se rendent quotidiennement au village voisin de Keur Ayib, où ils ont leur atelier, pour travailler et pour s'approvisionner, en cas de besoin, en produits d'élevage, le dimanche, jour de marché. Le cas de ce jeune ouvrier gambien est très représentatif de cet état de fait : 


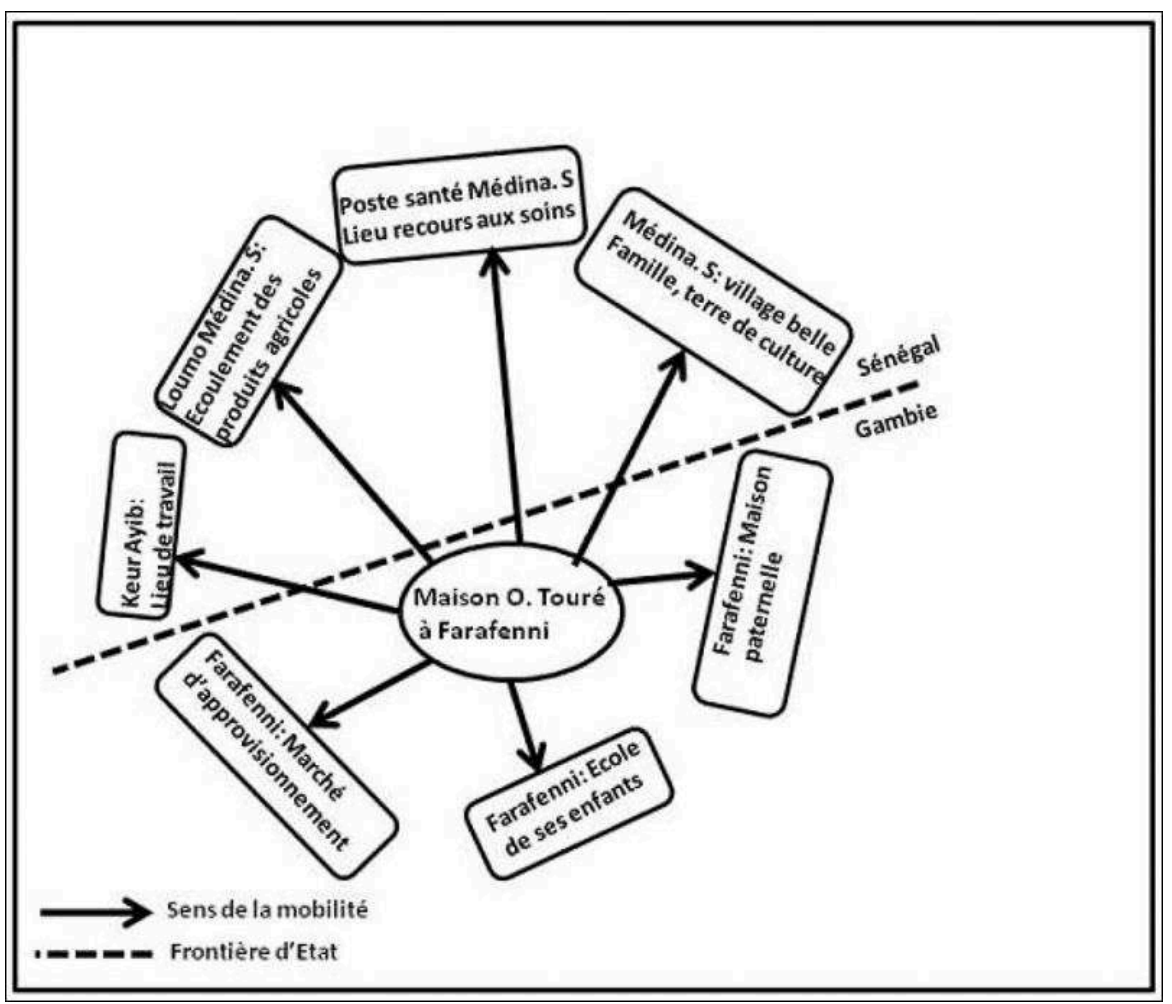

O. Touré, 37 ans, est né à Farafenni (Gambie) où il suivit pendant une dizaine d'années une formation en menuiserie métallique. En 2004, il ouvre un atelier à Keur Ayib (Sénégal) suite aux problèmes d'électricité que connait la ville gambienne où, cependant, il continue à loger. Dix-huit mois plus tard, il se marie avec une de ses cousines du village voisin de Médina Sabakh, où il a une parcelle de culture obtenue des mains de son beau-père et qu'il exploite chaque année. O. Touré, son épouse et le reste de sa famille se soignent uniquement au Poste de Santé de Médina Sabakh, qu'ils apprécient pour la qualité de ses soins, et le cas échéant au centre de santé de Nioro du Rip (Sénégal), chef-lieu de département.

Pour O. Touré comme pour d'autres, le territoire ne se limite pas à la ville de Farafenni, il intègre également les villages sénégalais frontaliers où se passe une partie de leur vie et où sont situés les aires où ils font paître leurs troupeaux, les loumo de Médina Sabakh et Keur Ayib, par exemple, où ils vont vendre leurs récoltes ou s'approvisionner en produits d'élevage. La situation est identique pour les populations sénégalaises frontalières. Les habitants de Pata n'ignorent pas, en effet, dans leur représentation territoriale, la proche ville gambienne de Brikama-Ba où ils ont des parents et où ils vont se soigner et s'approvisionner en denrées alimentaires et produits manufacturés. Il y a ainsi de véritables liens socio-spatiaux transfrontaliers. Les propos du Seyfo, ${ }^{5} \mathrm{du}$ district gambien d'Upper Badibou illustrent parfaitement l'existence d'un territoire transfrontalier : "Le Sénégal et la Gambie constituent le même peuple. Nous avons les même ethnies, nos coutumes, nos pratiques sociales sont identiques et nos familles sont les mêmes. Moi je suis gambien, chef du Upper Badibou, mais je me suis marié avec une femme sénégalaise originaire de Médina Sabakh. Cela prouve que nous sommes tous de la même famille. La plupart des habitants de cette zone (Upper Badibou) ont des familles et des biens de l'autre côté de la frontière et vice versa. A Médina Sabakh, je suis chez moi...».

Ces différents lieux fréquentés au quotidien constituent l'espace de vie des populations frontalières. Il y a un réel sentiment d'appartenance à ces espaces pour ces populations. 
Il s'agit certes d'espaces séparés par la frontière mais connectés spatialement par des réseaux d'itinéraires et caractérisés par une forte proximité sociale car habités par des populations unies par d'intenses liens socioculturels et religieux (confréries mouride et tidjane). La proximité géographique est également réelle entre ces espaces frontaliers reliés par des pistes rurales et/ou des routes bitumées. Par exemple, le temps de trajet en voiture entre Médina Sabakh et Farafenni varie entre 5 et 10 minutes; il est de 15 à 20 minutes entre Pata (Sénégal) et Brikama Ba (Gambie). Nous sommes donc en présence de territoires au sens donné par Joël Bonnemaison (1981), pour qui le territoire est « un ensemble de lieux hiérarchisés, connectés à un réseau d'itinéraires ». Le territoire est donc une somme de lieux reliés entre eux, un ensemble de lieux fréquentés, d'espaces vécus par un homme, un groupe social (Di Méo, 2001). Les populations frontalières de la Sénégambie, en fréquentant de par leurs mobilités divers lieux de part et d'autre de la frontière, mettent en place une structure spatiale constituée de lieux qui créent et forment le territoire. Ce dernier, loin de se clore comme son homologue politique (circonscrit par des frontières d'État), reste ouvert et prêt à intégrer d'autres lieux. Le territoire de la mobilité sociale est donc à géométrie variable.

\section{La production de territoires transfrontaliers par la circulation marchande}

La mobilité marchande elle aussi participe à la fabrication de territoires, car, selon Claude Raffestin (1980), « toute pratique spatiale induite par un système d'actions ou de comportements se traduit par une 'production territoriale' qui fait intervenir maillage, nœud et réseau». Le cas ci-dessous portant sur l'espace marchand de M. M’Baye, jeune commerçant sénégalais exerçant au marché central de Farafenni, en est un bel exemple. 


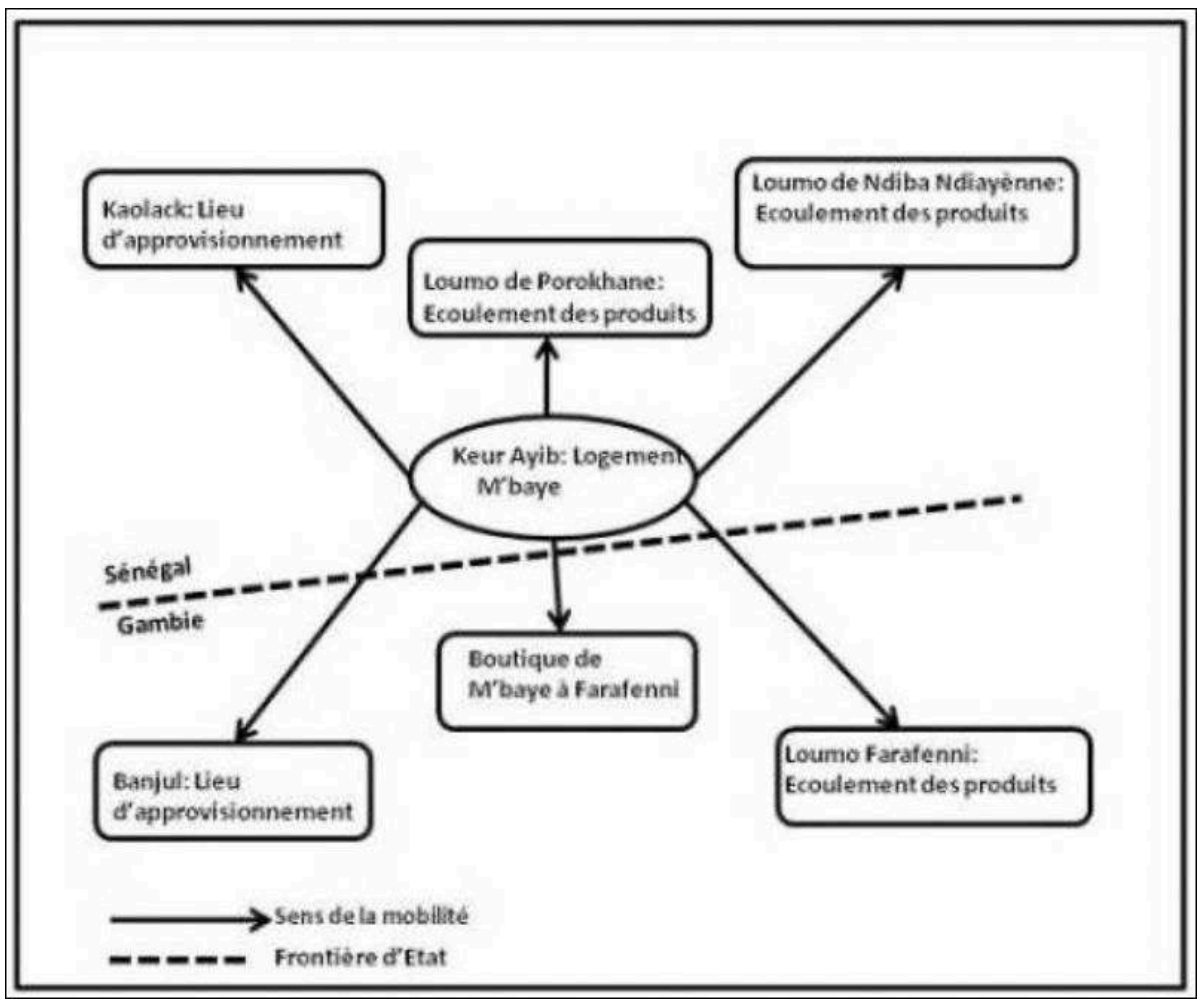

M. M'Baye est originaire de Porokhane dans la région de Kaolack. À 29 ans, il quitte son village natal où son commerce ne marchait plus et s'installe dans la ville commerciale de Farafenni où il se trouve avec l'aide d'anciens migrants sénégalais, une cantine au marché central de la ville. Par la suite, il trouve un logement à Keur Ayib tout en continuant d'exercer son activité à Farafenni qu'il regagne chaque matin après la nuit passée au village de Keur Ayib. M. M'Baye, comme d'autres commerçants sénégalais, guinéens et gambiens, s'approvisionne, selon les opportunités du marché, à Kaolack et à Banjul, et écoule ses produits manufacturés et alimentaires dans les loumo sénégalais frontaliers de Ndiba Ndiaye (le jeudi) et Porokhane (le lundi). Il fréquente également le loumo dominical de Farafenni.

L'espace marchand des acteurs du commerce transfrontalier en Sénégambie est donc constitué de places marchandes situées de part et d'autre des frontières, qui sont fréquentées quotidiennement. M. M’Baye et les autres commerçants considèrent, en effet, les lieux où ils s'approvisionnement et écoulent leurs produits comme faisant partie de leur espace de vie. Il existe ainsi un véritable sentiment d'appartenance et d'appropriation de cet espace marchand par ses acteurs. "Dans ce sens, on peut dire que l'espace marchand est un système territorial et qui produit un territoire » (Stary, 1995).

En définitive, l'analyse des mobilités transfrontalières révèle le caractère structurant de la frontière sénégalo-gambienne. L'effet-frontière crée des dynamiques locales qui renforcent les liens sociaux et spatiaux par-delà la frontière. La frontière est ainsi couture. Interface, la frontière Sénégal-Gambie est aussi discontinuité. Elle connaît en effet, mais rarement, des moments de fermeture, de renforcement des contrôles et des durées d'attente plus longues que d'habitude. Par exemple, des blocus sont souvent imposés par les transporteurs sénégalais pour contester les nombreuses tracasseries dont ils sont victimes de la part des forces de sécurité gambiennes lors de la traversée $\mathrm{du}$ bac de Farafenni. Cela conduit à un boycott du territoire gambien par les transporteurs sénégalais, entrainant de sérieuses perturbations sur la circulation des personnes et des biens. La mobilité des personnes sur la frontière connait également 
des difficultés avec le renforcement des contrôles relatifs à la circulation des produits notamment de la part des douaniers sénégalais dans le cadre de la lutte contre la fraude.

\section{La gouvernance des espaces partagés : l'épreuve de la mobilité}

Les territoires transfrontaliers de la frontière Sénégal-Gambie sont habités par des populations unies par de solides liens familiaux et qui utilisent les mêmes services publics. Les ressources naturelles de ces espaces font également l'objet d'une utilisation commune, comme les forêts de Diaroumé, Kandion, Pata, les pâturages de la Haute Casamance et les zones de culture. Se pose alors la question de la gouvernance transfrontalière au regard des enjeux que renferment ces territoires et des compétitions/conflits suscités.

\section{Les territoires transfrontaliers, des espaces de compétitions et de conflits}

41 Dans un contexte de forte croissance démographique et de migration de plus en plus intense ${ }^{6}$, la compétition pour l'accès et le contrôle des ressources devient de plus en plus âpre. Les ressources foncières et forestières deviennent, en Sénégambie et notamment, sur les espaces frontaliers sénégalo-gambiens, l'objet de fortes tensions entre acteurs (Sidibé, 2002, 2003 ; Enda Diapol, 2007). Dans les villages sénégalais de Dimiska, Darou Salam, Sinthiang Lathiery et Kéréwane (près de Pata), l'occupation des terres par les migrants gambiens a suscité de nombreux conflits avec les populations autochtones. Aussi, d'après les entretiens réalisés à Pata, beaucoup de Gambiens refusent-ils de rendre des terres que leur ont prêtées des paysans sénégalais et/ou cultivent au-delà de la frontière. Ces pratiques de paysans gambiens se traduisent par des conflits entre agriculteurs de part et d'autre de la frontière. La forêt de Pata illustre également un autre type de conflit foncier (Sidibé, 2002). Il s'agit des tensions vives entre migrants entrés dans la forêt de Pata dans le cadre de la culture arachidière et populations autochtones, ou même entre migrants, pour le contrôle de l'important potentiel foncier de la zone. Dans cette partie du Fouladou, où cohabitent foulacounda autochtones, migrants wolofs du Saloum et de la Gambie ainsi que peuls Fouta de la Guinée Conakry, les défrichements tous azimuts entraînent des conflits récurrents entre les différents acteurs. Par exemple, « le marabout fondateur du village de Médina Mandakh a été ouvertement défié par les migrants gambiens qu'il avait aidés à s'installer. Ces derniers qui se sont enrichis dans la culture arachidière, ne lui accordent plus aucune considération » (Sidibé, 2003). De plus, des conflits fonciers sont souvent notés entre villages frontaliers dans la zone de Kataba 1 et de Porokhane ${ }^{7}$ en raison de la non matérialisation de la frontière. Le conflit casamançais a aussi indirectement participé à l'émergence de conflits fonciers. L'installation massive de réfugiés casamançais dans les districts gambiens frontaliers de Foni Brefet, Foni Bintagn et Foni Kansala a suscité des tensions liées à l'occupation des terres. Cela a abouti à l'expulsion des réfugiés dans certains villages (Sifoe, Sitet, Bondali, etc).

Sur le plan des ressources forestières, les conflits sont récurrents entre exploitants forestiers gambiens et services étatiques sénégalais d'une part et populations locales 
d'autre part. À cela s'ajoutent les tensions entre agriculteurs sénégalais et éleveurs transhumants gambiens autour de la forêt de Pata du fait de la destruction des champs de culture.

Par ailleurs, dans une région où les équilibres géopolitiques sont instables (Diop, 1994), les populations sont parfois victimes de braquages, d'attaques à main armée, de viols perpétrés par des bandes armées qui tirent profit de la porosité des frontières et de la situation sécuritaire délétère. Ainsi, le conflit casamançais a favorisé le développement d'une importante activité d'exploitation frauduleuse de bois et d'autres ressources ligneuses des forêts de la Casamance (Sénégal) vers la Gambie (Enda Diapol, 2007; Diallo, 2014). Cette situation a également favorisé des vols fréquents de bétail le long des zones frontalières. Le témoignage du président du conseil Rural de Kataba 1 (Sénégal), Abdoulaye Badji, traduit bien l'ampleur du phénomène: "les habitants de Kataba 1 sont souvent victimes de vol de bétail. Avec l'insécurité et la proximité de la frontière, les voleurs entrent facilement en Gambie après leur forfait. Chaque année, je délivre plusieurs certificats de perte à des concitoyens qui ont perdu leur taureau. Car si le taureau est retrouvé en Gambie, les autorités demandent un certificat de perte avant de le restituer à son propriétaire»». Enfin, en dépit de son intensité, la mobilité des personnes se heurte souvent à des tracasseries, notamment lors de la traversée du fleuve Gambie; les autorités gambiennes procèdent parfois à des hausses, non justifiées, du prix de la traversée des véhicules et des personnes, notamment en période de crise diplomatique avec le Sénégal ou tout simplement pour augmenter les recettes du trésor gambien. Car la traversée du bac génère beaucoup de recettes pour l'État gambien. Les populations sénégalaises sont aussi victimes de tracasseries policières portant principalement sur la carte de séjour ou aliens card ${ }^{9}$ et les vérifications d'identité. Ces agissements des agents de sécurité gambiens s'expliquent selon plusieurs de nos interlocuteurs par la situation économique difficile que connaît le pays et par le bas niveau des salaires. Par ces opérations de contrôle, les policiers obtiennent illégalement de l'argent leur permettant de prendre en charge certains de leurs besoins quotidiens.

Ces différents exemples montrent tous les enjeux de la coopération transfrontalière impliquant les différents acteurs dans la gestion des ressources partagées et des problèmes communs.

\section{La coopération transfrontalière, entre initiatives locales et tensions étatiques}

Depuis le début des années 2000, des initiatives sont menées par les acteurs de part et d'autre de la frontière. La coopération est effective entre le district sanitaire de Nioro du Rip, dont dépend le poste de santé de Médina Sabakh, et le «Divisionnal Health Team » de Farafenni. Dans ce cadre, le poste de santé de Médina Sabakh organise avec l'hôpital de Farafenni des campagnes de vaccination communes et des échanges d'expérience (lutte conte les IST/SIDA, planning familial, etc). La coopération existe aussi dans le domaine de l'éducation et porte sur des jumelages entre établissements frontaliers (Lycée de Vélingara-High school of JanjanBureh) ou encore des échanges pédagogiques entre enseignants et de matériels didactiques (école Médina Sabakh 1Farafenni Proper). Dans ce territoire, la collaboration est également très forte entre les services de sécurité de Farafenni et ceux du poste frontalier de Keur Ayib. Ainsi, ces services s'assistent fréquemment pour la recherche de délinquants (auteurs de vols, 
d'agressions, etc) à travers des échanges d'informations ou encore la réalisation d'actions conjointes de traque et d'arrestation d'auteurs d'infractions. Sur la frontière Sud, les transporteurs de Vélingara (Sénégal) et ceux de Basse Santa Su (Gambie), dans leur volonté de faciliter les conditions de transport sur cet axe et de promouvoir la libre circulation des personnes et des biens ont mis en place, en décembre 2007, des dispositifs de concertation. Un cadre de concertation sur la libre circulation des personnes et des biens et un autre sur la gestion transfrontalière des ressources forestières ont été mis en place en 2009, respectivement dans la zone DiouloulouBrikama et Bounkiling-Kombo District. Ces espaces de dialogue qui regroupent différents acteurs sectoriels de part et d'autre de la frontière ont pour mission de promouvoir une gouvernance transfrontalière.

Par ailleurs, la coopération est effective entre villages frontaliers (Darou SalamAlloulaye, Pata-Tabanding, Hamdallaye-Sare Mamadou Fane, etc). Ces villages ont des champs collectifs, organisent périodiquement des campagnes de reboisement et mettent en place des pare-feu le long de la frontière. En outre, les populations de Sinthiang Koundara (Sénégal)-Niamanary (Gambie), en collaboration avec les élus locaux et les représentants des services étatiques, ont créé en 2007 un comité de vigilance qui a eu un rôle positif dans la lutte contre le vol de bétail. La mise en place du comité transfrontalier Diouloulou (Sénégal)-Brikama (Gambie) a été une grande réussite. Ce comité créé en 2004 grâce à l'appui de l'ONG Enda Diapol regroupe des élus locaux et des organisations de la société civile locale. Il a comme objectif de promouvoir l'intégration, la paix et le développement dans cette zone transfrontalière. Ainsi, le comité a mené plusieurs activités comme l'organisation d'une journée de reboisement, d'un festival culturel et de campagnes de sensibilisation sur la libre circulation des personnes et des biens. Il a aussi permis la réhabilitation de la piste DiouloulouKoudioubé-Brikama et celle entre Kabajo (Sénégal) et Kartung (Gambie).

Ces différents exemples de coopération mettent en évidence la diversité des acteurs de la gouvernance locale. Il s'agit principalement des services sectoriels de l'État (santé, éducation, sécurité), des organisations professionnelles (association d'élèves, de transporteurs, villageoises) et des autorités villageoises. La synergie entre ces différents acteurs s'explique par la proximité géographique et sociale (liens familiaux et socioculturels) ainsi que la similitude des problèmes de développement. Les acteurs locaux sont conscients de la nécessité de s'organiser et de gérer les problématiques de développement dans une perspective transfrontalière. Cependant, les initiatives actuelles de gouvernance transfrontalière mettent en évidence un jeu d'acteurs complexe. En effet, si dans les secteurs de la santé et de l'éducation les acteurs se complètent en s'apportant des appuis mutuels, dans les autres domaines les intérêts s'opposent parfois. Ainsi, les services gambiens bien qu'étant membre du cadre de concertation sur la libre circulation des personnes et des biens (Diouloullou-Brikama) ferment parfois les yeux sur les tracasseries dont sont victimes les populations sénégalaises. De plus, en dépit de l'existence d'un cadre de concertation des transporteurs de Vélingara-Bassa Santa $\mathrm{Su}$, les transporteurs sénégalais reprochent à leurs homologues gambiens de ne pas s'investir pleinement pour leur faciliter les conditions de transport en territoire gambien.

En plus, ici comme dans les autres territoires transfrontaliers sénégambiens, les relations entre les collectivités locales frontalières (conseil rural, municipal et conseil régional au Sénégal et comité de circonscription, conseil urbain et conseil régional en 
Gambie) sont inexistantes. Aucune forme de coopération directe n'existe entre elles. L'absence de relations inter-collectivités s'explique par la faiblesse de la décentralisation en Gambie, car en dépit de l'existence officielle d'un tel processus, l'autonomie des collectivités locales reste très limitée dans un pays marqué par la centralisation et le caractère militaire du régime. De ce fait, les autorités locales sont réservées vis-à-vis d'initiatives formelles de coopération transfrontalière avec leurs homologues sénégalais, surtout que des tensions diplomatiques sont souvent notées entre les deux États. La gouvernance transfrontalière inter-collectivités ne peut réussir si elle ne bénéficie de la bénédiction des États.

Pourtant, le fait que le Sénégal et la Gambie soient engagés dans des processus de décentralisation constitue en principe un atout pour la promotion de la coopération transfrontalière. De plus, les compétences transférées par les États aux collectivités territoriales dans le cadre de la décentralisation (santé, éducation, ressources naturelles, aménagement du territoire) sont identiques à celles conférées aux «paysfrontière " par la convention sur la coopération transfrontalière de la CEDEAO validée en 2006. À cela s'ajoutent les importants progrès réalisés avec notamment l'adoption, en 2008, du projet de directive entrant dans le cadre du processus d'institutionnalisation de la coopération transfrontalière dans l'espace CEDEAO.

Ce dispositif institutionnel offre donc un cadre favorable à la mise en œuvre d'initiatives formelles de coopération transfrontalière entre collectivités territoriales. Encore faudrait-il que les États inscrivent la coopération transfrontalière dans le dispositif juridique et institutionnel national à travers, par exemple, la prise en compte de la dimension transfrontalière dans les politiques de décentralisation et dans les commissions mixtes. Autrement dit, les États et les collectivités territoriales doivent inscrire la coopération transfrontalière dans le dispositif institutionnel et les pratiques de gouvernance locale des collectivités territoriales frontalières. Les accords de coopération et les traités signés ${ }^{10}$ entre le Sénégal et la Gambie sont favorables au renforcement des relations entre les deux États et au développement d'initiatives transfrontalières. Les décisions de la $6^{\mathrm{e}}$ session de la commission mixte Sénégal-Gambie tenue à Banjul (8 et 9 juillet 2013) s'inscrivent dans cette dynamique. Il a été décidé de construire le pont sur le fleuve Gambie, de diligenter la construction de postesfrontières juxtaposés (Keur Ayib/Keur Ali; Sénébo/Massira), d'organiser des patrouilles communes le long des frontières et de soutenir tous les projets de coopération sociale, culturelle, universitaire entre les deux pays.

51 Bien que les décisions prises soient pertinentes, que la plupart des accords soient ratifiés et que des relations diplomatiques existent avec notamment des réunions de la grande commission mixte Sénégal-Gambie et de la commission consultative, l'application des accords et conclusions reste difficile. Par exemple, les décisions prises lors de la $6^{\mathrm{e}}$ session de la commission mixte en 2013 , de la $4^{\mathrm{e}}$ commission consultative en 2011 et l'accord de sécurité de janvier 2010 (organisation de patrouilles mixtes) ne sont pas appliqués.

52 En réalité, les relations entre les deux États sont erratiques et achoppent souvent sur les intérêts nationaux. La Gambie, par exemple, n'a aucun intérêt à renforcer les patrouilles douanières et à mettre en place des postes frontaliers juxtaposés car la contrebande en direction du Sénégal est l'une de ses principales sources de devises. Il en est de même de la réalisation d'un pont au-dessus du fleuve Gambie. La situation actuelle offre à Banjul d'importantes recettes fiscales (paiement de droit de passage par 
les personnes et véhicules, taxes sur les commerçants) qui disparaîtraient avec la mise en place du pont sur ce fleuve. En revanche, la contrebande de produits alimentaires notamment (sucre, tomate, etc) constitue un manque à gagner et une sérieuse menace pour l'industrie sénégalaise tandis que la mise en place du pont sur le fleuve permettrait de faciliter davantage la mobilité entre le nord et le sud du Sénégal.

Ces divergences d'intérêts pèsent lourdement sur les relations entre les deux États. Elles ont d'ailleurs été à l'origine de la dissolution, en décembre 1989, de la Confédération de la Sénégambie créée en février 1982, suite à l'intervention de l'armée sénégalaise à Banjul, dans le cadre de l'opération Fodé Kaba II, pour restaurer le régime du président Dawda Jawara menacé par des révolutionnaires. Les tensions entre les deux pays sont récurrentes et résultent notamment de problèmes frontaliers (en 1969, 1974, 2008, 2009) consécutifs à l'absence et/ou à l'imprécision des limites territoriales. C'est pourquoi les deux États ont mis en place une commission conjointe de gestion des frontières qui s'inscrit dans le cadre du programme frontière de l'Union africaine (2007).

Par ailleurs, la complicité supposée ou réelle du pouvoir gambien avec la rébellion casamançaise est une grande source de tensions. Ainsi, la saisie d'un conteneur d'armes en provenance d'Iran à destination de Banjul, en octobre 2010, a entrainé une vive tension diplomatique entre le Sénégal et la Gambie car pour les autorités sénégalaises, les armes étaient destinées aux séparatistes de la Casamance. De plus, l'exécution de deux Sénégalais condamnés à morts en Gambie en août 2012, la hausse du tarif de la traversée du bac de Farafenni de 80 à $100 \%$ en 2005, l'arraisonnements intempestifs de bateaux de pêche sénégalais (en 2010, 2012, 2013) par les gardes côtes gambiennes et la décision unilatérale du président gambien de fermer la frontière le 19 avril 2014 sont autant d'actes qui contribuent à la dégradation des relations diplomatiques. Il faut noter que la Gambie reproche depuis 2010 au Sénégal d'abriter des opposants à son régime et accueille mal les mesures de répression contre la fraude prises par le gouvernement sénégalais.

Ces tensions entre les deux États constituent une limite pour l'institutionnalisation et le renforcement des initiatives locales de coopération transfrontalière.

\section{Conclusion}

Ouverte et très poreuse, la frontière Sénégal-Gambie est le lieu d'une mobilité particulièrement intense. Celle-ci est l'œuvre d'acteurs socioéconomiques et marchands qui, s'appuyant sur les liens parentaux, exploitent les disparités et opportunités nées de l'effet-frontière. Ces acteurs finissent, à travers leurs diverses mobilités quotidiennes, par connecter divers lieux situés de part et d'autre de la frontière, créant ainsi des territoires transfrontaliers, espaces de vie vécus et appropriés.

Espaces partagés et de fortes solidarités intercommunautaires, ces territoires transfrontaliers connaissent néanmoins des compétitions et conflits entre divers acteurs de part et d'autre de la frontière. Depuis le début des années 2000, de nombreuses initiatives de coopération portées par les acteurs locaux œuvrent pour le renforcement de l'intégration et la consolidation de la paix dans ces territoires. Cette coopération transfrontalière bénéficie d'un cadre institutionnel favorable avec la décentralisation en cours au Sénégal et en Gambie et d'autre part, des progrès 
institutionnels de la CEDEAO en matière de coopération transfrontalière. Elle peut cependant être freinée par les tensions diplomatiques et les divergences d'intérêts entre les deux États. Le renforcement des initiatives locales et l'institutionnalisation de la coopération transfrontalière dépendront fortement de l'engagement et d'une réelle implication des États. De ce fait, la problématique de la gouvernance transfrontière en Sénégambie mérite d'être davantage documentée en insistant notamment sur le rôle des États et le rapport de pouvoir entre les acteurs locaux, publics et privés, formels et informels.

\section{BIBLIOGRAPHIE}

Bach D., 1998. Régionalisation, mondialisation et fragmentation en Afrique Subsaharienne. Karthala, Paris, $320 \mathrm{p}$.

Badié B., 1995. La fin des territoires. Essai sur le désordre international. Fayard, Paris, 278 p.

Barry B., 1988. La Sénégambie du XV $V^{e}$ au XIXe siècle, traite négrière, islam et conquête coloniale. Paris, L'Harmattan, $430 \mathrm{p}$.

Bennafla K., 2002. Le commerce frontalier en Afrique Centrale : Acteurs, espaces, pratiques. Karthala, Paris, $366 \mathrm{p}$.

Bonnemaison J., 1981. Voyage autour du territoire. L'espace géographique, n 4, p. 249-262.

Diallo M-M., 2014. Frontières, stratégies d'acteurs et territorialités en Sénégambie: cas des frontières Sénégal-Gambie et Sénégal-Guinée Conakry. Thèse de Doctorat géographie-aménagement, Université Paul Valéry Montpellier, $540 \mathrm{p}$.

Di Méo G., 2001. Géographie sociale et territoires. Nathan, Paris, 320 p.

Diop M-C., 1994. Le Sénégal et ses voisins. Sociétés-espaces-temps, 325 p.

Dubresson A., Raison J-P., 2003. L'Afrique Subsaharienne, une géographie du changement. Armand Colin, Paris, 245 p.

Enda Diapol, 2007. Les dynamiques transfrontalières en Afrique de l'Ouest. CRDI/Enda Diapol/ Karthala, 219 p.

Fanchette S., 2001. Désengagement de l'État et recomposition d'un espace d'échange transfrontalier : la Haute Casamance et ses voisins. Revue Autrepart, ${ }^{\circ}{ }^{\circ}$ 19, p. 91-113.

Foucher M., 2014. Frontières d'Afrique. Pour en finir avec un mythe. Paris, CNRS éditions, $64 \mathrm{p}$. Igué J-O., 1995. Le territoire et l'État en Afrique : Les dimensions spatiales du développement. Karthala, Paris, $277 \mathrm{p}$.

Kane A.F., 2010. Les frontières et leurs conséquences en Afrique de l'Ouest de la Mauritanie à la Guinée Conakry: Espace mauritano-guinéen. Thèse de doctorat d'État, Université Cheikh Anta Diop de Dakar, 4743 p + annexes. 
Lefebvre C., 2015. Frontières de sable, frontières de papier. Histoire de territoires et de frontières, du jihad de Sokoto à la colonisation française du Niger, XIXe-XXe siècles. Paris, Publications de la Sorbonne, $543 \mathrm{p}$.

Meagher K., 2003. L'antichambre de la globalisation? Ajustement structurel, globalisation et commerce transfrontalier en Afrique de l'Ouest. Wabi, $25 \mathrm{p}$.

Ninot O., Lesourd M., Lombard J., 2002. Nouveaux espaces, nouvelles centralités: échanges et réseaux en milieu rural sénégalais. Historiens et géographes, n 379, p. 141-152.

Raffestin C., 1980. Pour une géographie du pouvoir. Paris, Librairies techniques, 249 p.

Roitman J., 2003. La garnison-entrepôt : une manière de gouverner dans le bassin du lac Tchad. Critique internationale $2 / 2003, \mathrm{n}^{\circ} 19, \mathrm{p} .93-115$. www.cairn.info/revue-critiqueinternationale-2003-2-page-93.htm. DOI : 10.3917/crii.019.0093

Sidibé M., 2003. Les migrants saloum-saloum de la forêt classée de Pata (Sénégal). De l'installation différentielle au redéploiement spatio-économique. In Lesourd M. (coord.), L'Afrique. Vulnérabilité et défis. Nantes, Éditions du Temps, Collection Questions de géographie, p. 199-218.

Sidibé M., 2002. Entre le Saloum et la forêt de Pata : mobilité des migrants, intégration des espaces. Revue européenne des migrations internationales, vol. $18, \mathrm{n}^{\circ} 2$, Migrations et environnement, p. 189-207.

Simon-Loriere H., Lysaniuk B., 2015. La diffusion d'Ébola dans les pays de la Mano River : approche géographique. EchoGéo [En ligne], Sur le Vif, mis en ligne le 02 février 2015. http:// echogeo.revues.org/14096 ; DOI : 10.4000/echogeo.14096

Stary B., 1995. Réseaux marchands et territoires étatiques en Afrique de l'Ouest. Communication au colloque Le territoire, lien ou frontière, Paris, 2-5 octobre 1995, 10 p.

Walther O., 2008. Affaires de patrons. Villes et commerce transfrontalier au Sahel. Berne, Peter Lang, 478 p.

Walther O., 2007. Villes-frontières et réseaux informels sahéliens (Gaya-Malanville-Kamba). Geographica Helvetica, ${ }^{\circ}$ 1- 07, p. 33-42.

Walther O., Rétaillé D., 2008. Le modèle sahélien de la circulation, de la mobilité et de l'incertitude sapatiale. Autrepart, 3/2008, n 47, p. 109-124.

\section{NOTES}

1. Au sens de l'intégration régionale en cours dans l'espace ouest africain (UEMOA et CEDEAO).

2. Il faut préciser que bien avant la mise en place des frontières coloniales, plusieurs groupes ethniques furent séparés par des frontières historiques dans le cadre de royaumes et d'entités politiques. Par exemple, les frontières du royaume de Sokoto séparées déjà les populations haoussa, touareg (Lefebvre, 2015).

3. La frontière Sénégal-Gambie ( $740 \mathrm{~km}$ de long) compte 14 postes-frontières (en raison de 7 par pays). La multiplicité des passages frontaliers traduit l'intensité des mobilités, surtout marchandes, dans les zones bordières sénégambiennes.

4. Marché hebdomadaire en langue locale peule.

5. Le Seyfo est le chef de district en Gambie. Il est le représentant de l'État dans le district et travaille sous l'autorité du gouverneur de région. Il est, dans certains cas, nommé par le gouvernement central et dans d'autres élu par les populations locales. Il faut noter que le district est, en Gambie, le second niveau de la hiérarchie administrative et territoriale après la région. 
6. La croissance démographique est supérieure à $2 \%$ par an en Sénégambie. En Gambie, elle est de $5 \%$ pour la région de Brikama, Mansakonko (6,1\%), Kerewan $(5,8 \%)$, Kuntaur (6\%) et Janjanbureh (6,2\%). Au Sénégal, le taux de croissance annuel est de 2,6\% pour Ziguinchor et $2,5 \%$ pour Kolda. Pour ce qui est de la migration elle s'explique par la disponibilité de ressources foncières et forestières ainsi que le développement d'activités marchandes notamment dans les zones frontalières. Elle se fait des campagnes vers les villes, entre villes et entre campagnes (agriculteurs et exploitants forestiers Guinéens, Gambiens et Sénégalais du nord qui migrent vers les terres et forets fertiles du sud Sénégal, etc).

7. Dans la zone de Porokhane (Sénégal)-Minte Kounda (Gambie), les conflits fonciers ont été fréquents notamment en 2008 entre villages frontaliers et ont conduit à l'intervention du souspréfet sénégalais de Paoskoto. Sur la frontière sud, l'occupation par des Gambiens de la localité sénégalaise de Touba (communauté rurale Kataba 1) a suscité de vives tensions entre populations frontalières, entrainant l'envoi par la présidence de la république du Sénégal d'une délégation qui a initié une médiation avec les autorités gambiennes.

8. Entretien le mercredi 22 décembre 2010.

9. Il s'agit d'une taxe annuelle de $20000 \mathrm{Fcfa}$, que doit payer toute personne de nationalité étrangère âgée de 18 ans et plus et résidant en territoire gambien.

10. Arnold Hughes (voir Diop, 1994) note qu'entre 1965, année d'indépendance de la Gambie, et 1982, trente (30) traités de coopération ont été signés entre les deux États. Les plus importants sont le Traité d'association de 1965, le Traité économique de 1970 et la création de l'OMVG en 1978. Les autres accords portent sur la sécurité et la défense, la construction de routes et le transport par voie terrestre, l'agriculture, la fraude, etc.

\section{RÉSUMÉS}

Cet article se propose d'étudier le rôle de la frontière dans la production quotidienne de territoires. Discontinuité géographique majeure, la frontière est traditionnellement définie comme la limite d'un territoire d'État, symbole de sa souveraineté. La frontière Sénégal-Gambie qui garde cette fonction, créée de nombreux effets-frontières largement exploités par les populations. Les acteurs sociaux et marchands des zones frontalières sénégalo-gambiennes, à travers leurs mobilités quotidiennes consécutives à l'effet-frontière connectent des lieux de part et d'autre de la frontière. Ils produisent ainsi des territoires, espaces vécus et appropriés. La frontière est ici lien et couture.

Espaces partagés et de fortes solidarités intercommunautaires, ces territoires transfrontaliers sont cependant l'objet de compétitions et de conflits qui sont aujourd'hui pris en charge par les acteurs locaux à travers diverses initiatives de coopération transfrontalière. Le renforcement de ces initiatives locales, voire leur formalisation, bénéficie d'une part du processus de décentralisation en cours au Sénégal et en Gambie, et d'autre part, des progrès réalisés récemment par la CEDEAO dans le cadre de la formalisation de la coopération transfrontalière. Il pourrait cependant être handicapé par les divergences d'intérêts et les tensions entre les deux États.

This article aims to study the role of the border in fashioning daily activity territories. A major geographical discontinuity, the border is traditionally defined as the limit of a state territory, and a symbol of its sovereignty. The Senegal-Gambia border performs this function, and creates 
numerous border effects that are exploited by the population. Social actors and merchants in the Senegal-Gambia border areas, through their daily mobility around the border, connect places on both sides of that border. In so doing, they produce distinct territories, experiences and spaces. The border is a link that joins and binds together shared spaces and strong communal solidarity. But these border territories are also subject to competition and even to conflict relating to local actors involved in various border cooperation initiatives. The strengthening or formalization of these local initiatives involves part of the ongoing decentralization process in Senegal and Gambia, and also reflects recent progress made by ECOWAS in the formalization of border cooperation. This could, however, be hampered by differences of interest and by tensions between the two states.

INDEX

Keywords : border, territory, mobility, cross-border, Senegal, Gambia, Senegambia Mots-clés : frontière, territoire, mobilité, coopération transfrontalière, Sénégal, Gambie, Sénégambie

\section{AUTEUR}

\section{MOHAMADOU MOUNTAGA DIALLO}

Mohamadou Mountaga Diallo, mountaga2006@yahoo.fr ou mountaga@geradsn.org, est Docteur en géographie-aménagement, Chercheur au GERAD/Université de Dakar et membre associé au GRED/Université Montpellier 3 